\title{
A Novel Pixel Design for AM-OLED Displays Using Nanocrystalline Silicon TFTs
}

\author{
Chen-Wei Lin, Mango C.-T. Chao, Member, IEEE, and Yen-Shih Huang
}

\begin{abstract}
This paper presents a novel pixel design for active matrix organic light emitting diode (AM-OLED) displays using nanocrystalline silicon thin-film transistors (TFTs). The proposed pixel design can effectively reduce the variation of its stored display data caused by the leakage current of nanocrystalline silicon TFTs, which can in turn increase the contrast resolution of AM-OLED displays. With a proper setting of its capacitors, the proposed pixel design can achieve a $5.55 \times$ reduction on its display-data variation while requiring only a $1.15 \times$ write time when compared to the typical pixel design. The aperture ratio resulting from the layout of the proposed pixel design can also be maintained above $40 \%$, which satisfies the specification of most AM-OLED displays. A series of simulations as well as measurement results are provided to validate the effectiveness of the proposed pixel design.
\end{abstract}

Index Terms-Active Matrix Organic Light Emitting Diode (AM-OLED), coupling effect, microcrystalline thin-film transistor (TFT), nanocrystalline TFT.

\section{INTRODUCTION}

D URING the past few years, major display manufacturers have put a lot research efforts into the area of active matrix organic light emitting diode (AM-OLED) displays, which use self-light-emitting devices, require no backlight elements, and in turn are lighter, thinner, and less power-consumed compared to the current display mainstream, active matrix liquid crystal display (AM-LCD displays) [1], [2]. AM-OLED display can also provide faster response time, wider viewing angle, and better front-of-screen (FOS) quality, and hence is considered as the best candidate for next-generation displays [1], [2]. In order to build a robust backplane which can reliably control OLED's current and thus its lightness, a stable and high-quality thin-film transistor (TFT) technology is required. However, the available TFT technologies in current industry are not yet cost-effective or stable enough, which has been the biggest barrier for developing quality AM-OLED displays today [1], [3].

Table I compares the three major prospects of the TFT technology used for building the backplane of AM-OLED displays, which are: 1) amorphous silicon ( $\alpha$-Si) TFT; 2) polycrystalline silicon (poly-Si) TFT; and 3) nanocrystalline silicon (nc-Si) TFT (or microcrystalline silicon TFT). The first prospect $\alpha$-Si

Manuscript received September 16, 2009; revised December 23, 2009. First published March 11, 2010; current version published May 25, 2011.

C.-W. Lin and M. C.-T. Chao are with the Department of Electronics Engineering and Institute of Electronics, National Chiao Tung University, Hsinchu 30010, Taiwan (e-mail: eeer.ee97g@nctu.edu.tw; mango@ faculty.nctu.edu.tw).

Y.-S. Huang is with the Industrial Technology Research Institute, 310 Taiwan (e-mail: oled@itri.org.tw).

Digital Object Identifier 10.1109/TVLSI.2010.2042735
TABLE I

COMPARISON OF SILICON-BASED TFTS

\begin{tabular}{|c||c|c|c|}
\hline Materials & $\alpha-\mathrm{Si}$ & poly-Si & nc-Si \\
\hline Effective Mobility & Low & High & Medium \\
\hline Reliability & Poor & Excellent & Good \\
\hline Process Temperature & $<300^{\circ} \mathrm{C}$ & $450^{\circ} \mathrm{C}$ & $<300^{\circ} \mathrm{C}$ \\
\hline Electric Uniformity & Excellent & Poor & Good \\
\hline Leakage Current & Low & High & Medium \\
\hline
\end{tabular}

TFT, which is currently used for building the backplane of AM-LCD displays, has the advantages of low leakage, low process temperature, better electric uniformity, and easy deposition, resulting a relatively low manufacturing cost [1], [2], [4]. However, $\alpha$-Si TFT suffers from its low mobility, which may result in a low aperture ratio and in turn low illumination efficiency. $\alpha$-Si TFT also suffers from its large threshold-voltage shift over time, which significantly reduces the reliability of AM-OLED displays since a large current needs to pass through the TFTs which drive the OLEDs. Such an OLED-driving TFT is not required in AM-LCD displays, whose light source comes from a stable backlight element.

In contrast with $\alpha$-Si TFT, the second prospect poly-Si TFT, which is also a mature technology in current industry, can provide stable threshold voltage over time and high mobility for driving OLED due to its larger silicon grain size [1], [2]. However, by using the technique of excimer laser annealing for crystallization, poly-Si TFT has the disadvantages of low electric uniformity, which limits the application of poly-Si TFT only on small-size panels [1], [2]. Also, the overall manufacturing cost of poly-Si TFT is high because of its high process complexity, the required laser equipments, and its high process temperature.

The third prospect nc-Si TFT, whose silicon grain size is between the other two TFT technologies, can overcome the drawbacks of low mobility and poor reliability when compared to $\alpha$-Si TFT, and at the same time overcome the drawbacks of high manufacturing cost and poor electric uniformity when compared to poly-Si TFT. As in [1], [4]-[6], nc-Si TFT can be manufactured by using the conventional $13.56 \mathrm{MHz}$ plasma-enhanced chemical vapor deposition (PECVD), whose manufacturing infrastructures are already well established in current industry for $\alpha$-Si TFT technologies. Furthermore, if the nc-Si TFT is constructed by using the bottom-gate structure [1], [3] instead of the top-gate structure [5], [6], the process flow can match the one used for the current manufacturing of AM-LCD displays. In this condition, the technology of nc-Si TFT can reuse the current production lines of AM-LCD displays and, thus, can be brought to mass production sooner and more economically. 
Overall, the nc-Si TFT combines several advantages of both poly-Si and $\alpha$-Si TFTs, is cost-effective in manufacturing, and hence emerges as a promising TFT technology for AM-OLED displays. However, the biggest barrier preventing the nc-Si TFT from production is its high drain-source turn-off current (leakage current). When the typical pixel design is used, this high leakage current may result in either a low contrast resolution or the lose of a stored display data before an image frame is refreshed. A high leakage current is a common drawback of using the PECVD [1], [5] and may become worse when the bottom-gate structure is used [1], [3], [5]. Several previous works attempted to reduce this leakage current from the aspects of changing TFT's structures [5], [6], material [1], or deposition mechanisms [7], but yet the current progress along this research line is still not sufficient enough to reach the standard for production.

In this paper, we attempt to push the use of nc-Si TFTs into production by proposing a novel pixel design of AM-OLED displays. The proposed pixel design can effectively reduce the degree of display-data variation caused by nc-Si TFT's leakage. Also, its write-time overhead and area overhead can be properly limited by the choice of its capacitance setting and layout techniques, respectively. The analysis and simulation for both display-data variation and data write time will then be provided. An optimal configuration of the proposed pixel design can be obtained through proper simulation. The measurement result based on a nc-Si TFT technology developed by ITRI [8] further confirms our analysis and simulation for the proposed pixel design. In general, the contrast resolution saved by the proposed pixel design can be up to more 2 digital bits, which significantly ease the pressure of finding a new low-leakage material or deposition mechanism, and in turn speed up the production of AM-OLED displays using nc-Si TFTs.

\section{BACKGROUND OF AM-OLED DISPLAYS AND THE TYPICAL PIXEL DESIGN}

Unlike AM-LCD panels, which need a backlight element to be its light source, AM-OLED panels use the OLED thin film embedded in its multilayer structure for illumination and hence requires no backlight element. Fig. 1 illustrates the typical multilayer structure used for an AM-OLED panel, which consists of the following five layers listed from bottom to top: 1) substrate layer; 2) control-circuit layer; 3 ) illuminant layer; 4) cathode layer; and 5) cover layer. The substrate layer and cover layer are usually made by glass, which allows the light passing through, isolates the other inner layers from water and oxygen, and hence prevents the aging of its organic material as well as the change of TFT's electrical characteristics. The control-circuits layer utilizes TFTs and capacitors to form a pixel array, which can control the degree of illumination of each OLED, which is evaporated on the illuminant layer accordingly. The cathode layer is made by metal $\mathrm{Al}$ and used as the ground signal connected to OLED.

As a result of the multilayer structure shown in Fig. 1, the panel's images are observed from the bottom of the panel since the layer on top of the illuminant layer (made by aluminum) is opaque. Thus, the light generated by the OLEDs needs to pass through the control-circuit layer, and which in turn needs to provide a transparent region on each pixel design as a channel for

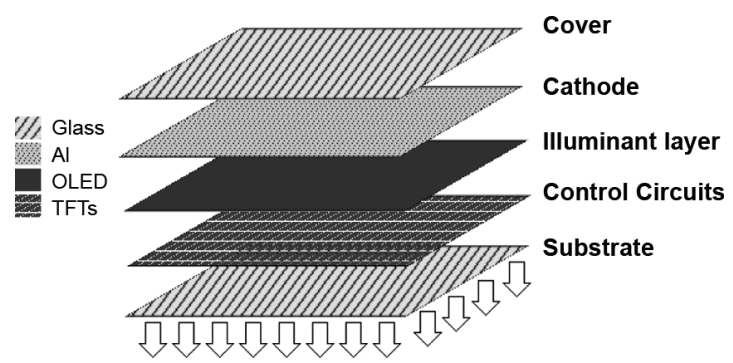

Fig. 1. Typical multilayer structure of an AM-OLED panel.

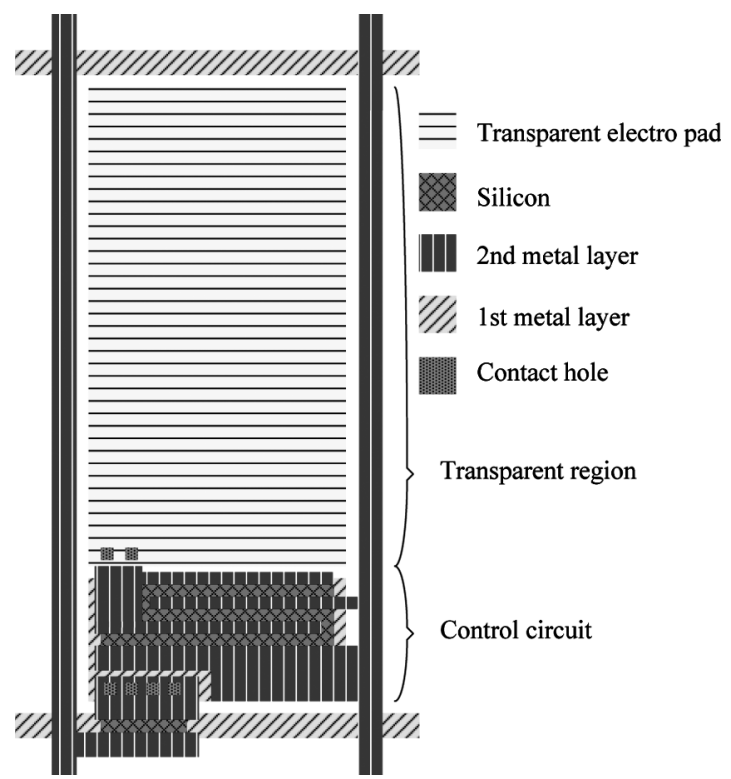

Fig. 2. Layout of a typical pixel design and its resulting transparent region.

light. The transparent region is made by a transparent electrical conductor, indium tin oxide (ITO), and is used as an electropad connecting to the OLED. Fig. 2 shows a typical pixel layout with the corresponding transparent region. Based on the OLED technologies used in our design, the ratio of the transparent region in a pixel design (defined as aperture ratio) have to be more than $40 \%$ of the pixel's total area to provide sufficient luminance under the constraint of current density.

Following is the calculation of the minimum aperture ratio. First, we use (1) to calculate the required current passing through the OLED (denoted as $I_{\mathrm{OLED}}$ ) such that the desired luminance of a panel (denoted as Lum) can be provided. In (1), the luminance of the panel ( Lum) is set to 200 nits $\left(1 \mathrm{nit}=1 \mathrm{Cd} / \mathrm{m}^{2}\right)$, which accords to the specification of a quality AM-OLED panel [9]. The pixel area (denoted as Area) in a 4.1" QVGA AM-OLED panel is $86.78 \times 260.35 \mu \mathrm{m}^{2}$. The luminance efficiency (denoted as $L E$ ) of the OLED in use is set to $4 \mathrm{Cd} / \mathrm{A}$, which is a relatively weak OLED in current OLED market. The panel transmittance (denoted as $P T$ ) is set to 0.8 , which is determined by the transmittance of the transparent electropad (ITO) and the substrate (glass). By solving (1) with the above parameters, the required current $\left(I_{\mathrm{OLED}}\right)$ is equal to $1.4 \mu \mathrm{A}$ 


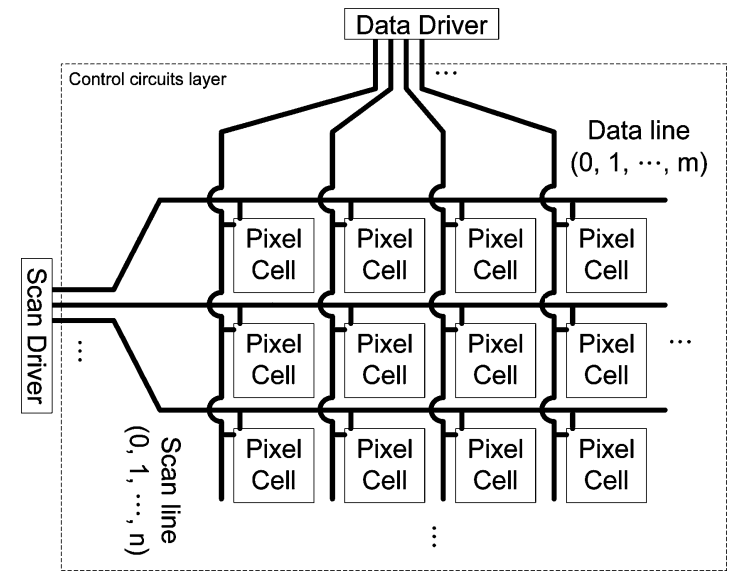

Fig. 3. Overview of a pixel array.

The aperture ratio of a pixel design (denoted as $A R$ ) determines the area of the transparent electropad, i.e., the area of the evaporated OLED. The area of the evaporated OLED further determines the current density of the OLED [10], [11]. An excessive current density may significantly degrade the reliability and lifetime of the OLED. In the following calculation, we set the upper bound of the current density (denoted as CDen) to $20 \mathrm{~mA} / \mathrm{cm}^{2}$ as suggested by [10], [11]. By solving (2), the aperture ratio needs to be larger than $31 \%$. Thus, setting the minimum aperture ratio to $40 \%$ is actually a high standard for current panels and can already cover a significant range of design margins

$$
\frac{I_{\mathrm{OLED}}}{\mathrm{Area} \cdot A R} \leq \mathrm{CDen} \text {. }
$$

Fig. 3 shows an overview of an $m \times n$ AM-OLED pixel array locating on the control-circuit layer, where the lightness of a pixel is controlled by the data stored in a pixel cell. In this pixel array, a row of pixel cells is selected by the signal Scan_line. The data of pixel cells on a selected row is fed through the signal Data_line. The signal Scan_line and Data_line are controlled by an outside driving IC through a runner, respectively. Such an AM-OLED pixel array is similar to a DRAM array except the following differences: 1) the data stored in each AM-OLED pixel cell is an analog signal rather than a digital signal; 2) an AM-OLED array only performs write operations while a DRAM array performs both read and write operations; and 3) the write operation of an AM-OLED pixel array is performed in a row basis serially and ceaselessly while DRAM cells can be accessed randomly.

Fig. 4 illustrates the typical pixel design used in current AM-OLED displays, which consists of two TFTs, one capacitor, and one OLED. As shown in Fig. 4, the lightness of an OLED is determined by the current flowing through the TFT $M_{2}$, which is further controlled by the display data stored in the capacitor $C_{s t}$. The TFT $M_{1}$ functions as a switch for determining whether the data from Data_line should be written in the capacitor $C_{s t}$ according to signal of $S$ can_line. With this pixel design, the electric signal stored in the capacitor $C_{s t}$ can be transformed into an optical signal displaying on an OLED.

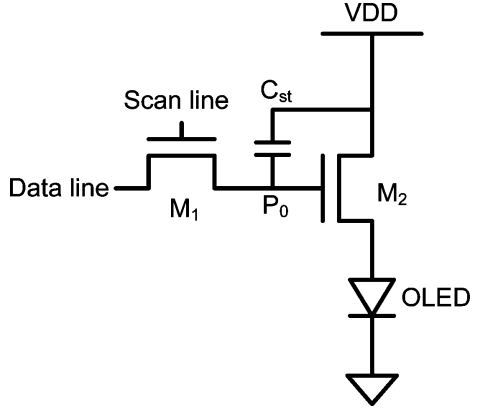

Fig. 4. Schematic of the typical pixel design.

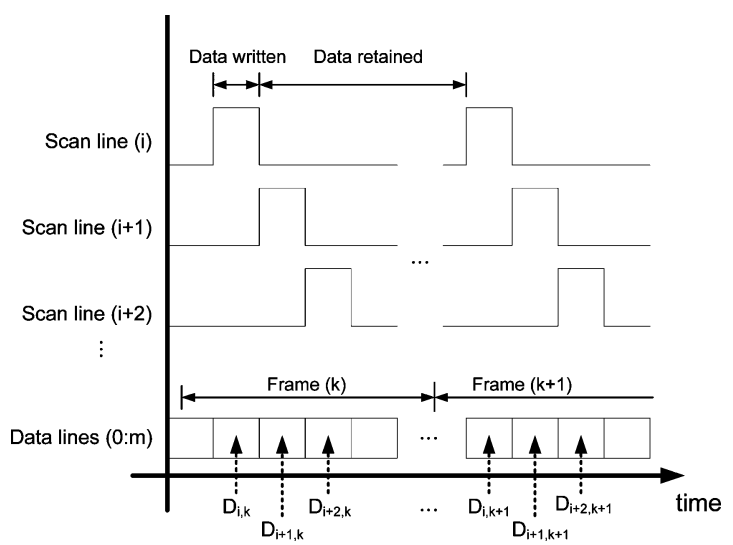

Fig. 5. Exemplary timing diagram of Scanline and Dataline for an AM-OLED pixel array.

Fig. 5 shows an exemplary timing diagram of Scan_line and Data_line on an AM-OLED pixel array. The signals on Scan_line are sequential and non-overlapped pulses. The signal on Data_line represents the image data to be displayed on a pixel row and is notated by $D_{i, k}$, where $i$ and $k$ represent the index of the corresponding pixel row and time frame, respectively. When Scan_line on a row is turned on, the corresponding pixel cells enter the "data-written" phase and their display data is written in. When Scan_line on the row is turned off, the corresponding pixel cells enter the "data-retained" phase and the pixel cells need to maintain the stored data until the next write operation on the current row. Thus, the time of an image frame is equal to the total time of a data-written phase and a data-retained phase, which is actually equal to the total number of rows times the time of a data-written phase.

Therefore, the quality of a proposed pixel design can be determined by its required time of writing in a display data and its capability of retaining a display data. The time of an image frame is fixed and usually set to $16.7 \mathrm{~ms}$ (reciprocal of $60 \mathrm{~Hz}$ ) according to the sensitivity of human's vision. Thus, the time of a data-written phase determines the number of pixel rows to be supported in a display, i.e., the size of the display. The capability of retaining a display data affects the degree of display-data variation during the data-retained phase, and in turn determines the contrast resolution represented by its least significant bit. Also, the proposed pixel design has to be subject to the pixel-area limit of a display panel and the required aperture ratio. 


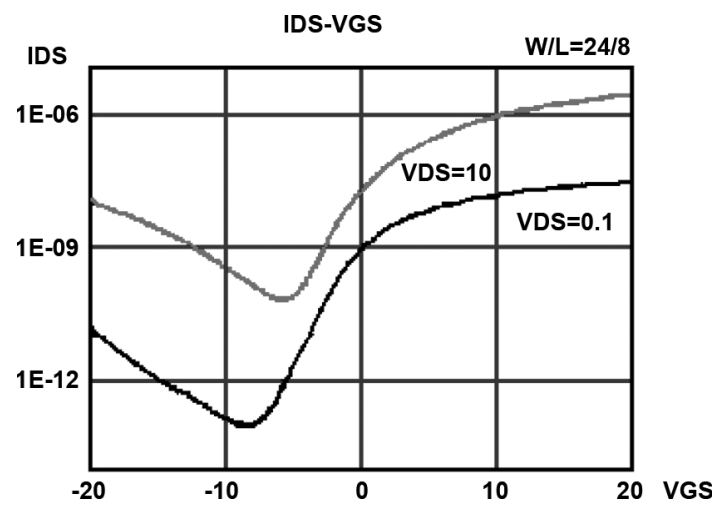

Fig. 6. $I_{d s}$ versus $V_{g s}$ of a nc-Si TFT [1].

\section{Challenge OF USING NC-Si TFTS IN AM-OLED DISPLAYS}

\section{A. Problem: High Leakage Current of nc-Si TFTs}

Despite of providing several advantages on its mobility, reliability, and manufacturing cost, the nc-Si TFTs suffers from its large turn-off leakage current [1], [3], [5], [12], which does not exist for $\alpha$-Si TFTs or can be solved by using lightly-dopeddrain (LDD) technique for low-temperature-polysilicon TFTs, which is a poly-Si TFT technology [2]. Fig. 6 shows the drainsource current $I_{d s}$ of a nc-Si TFT versus gate-source voltage $V_{g s}$ in condition of different drain-source voltage $V_{d s}$ [1]. As Fig. 6 shows, $I_{d s}$ of a nc-Si TFT may significantly increase when $V_{g s}$ is smaller than a certain amount. This characteristic of large turn-off leakage current $\left(I_{\text {off }}\right)$ results in that the switch TFT $M_{1}$ may not be able to cut off the connection between $P_{0}$ and Data_line (as shown in Fig. 4) during the data-retained phase. Thus, the data stored on $C_{s t}$ would be interfered by the data at Data_line writing into the other pixel cells in the same column, which may result in significant display-data variation during the data-retained phase.

\section{B. Straightforward Solutions}

For the engineers who cannot acquire better TFT devices at present, the following two straightforward solutions can be applied to the typical pixel design to minimize the display-data variation resulting from nc-Si TFT's large leakage current. The first solution is to increase the refresh rate. The second one is to increase the size of the capacitor $C_{s t}$ or the channel length of the TFT $M_{1}$.

1) Higher Refresh Rate: The objective of increasing refresh rate is to reduce the time of the data-retained phase such that the variation of the stored voltage of $C_{s t}$ can also be reduced before the new display data is written in. However, increasing the refresh rate may result in the following three drawbacks. First, increasing the refresh rate needs a stricter specification to both the data-driver IC and the scan-driver IC, which may in turn increase the design efforts and the cost of both driver ICs. Second, increasing the refresh rate requires a higher power consumption of the display, which may prevent the AM-OLED displays from mobile applications. Last and most importantly, the time for writing in display data to a pixel cell needs to be reduced since a frame period is equal to the total number of pixel rows times the time of the data-written phase. Otherwise, the number of affordable pixel rows become smaller. The time of writing in a display data is determined by the mobility and the channel width of the switch TFT $M_{1}$. However, increasing the mobility of a TFT may not be always achievable and increasing the channel width of the switch TFT may increase its leakage at the same time, which contradicts with our original objective of using a higher refresh rate.

2) Larger $C_{s t}$ or Longer Channel Length of $M_{1}$ : This circuit-level solution focuses on increasing the $R C$ time constant between $C_{s t}$ and $M_{1}$, where $C$ and $R$ represent the capacitance of $C_{s t}$ and the drain-source resistance of $M_{1}$ during the data-retained phase (which is also denoted as $R_{M_{1-o f f}}$ ). Usually, increasing $C_{s t}$ is not preferable because the layout area of $C_{s t}$ already occupies a significant portion of the whole pixel design. Doubling the size of $C_{s t}$ may significantly decrease the aperture ratio of the pixel design. Also, increasing the size of $C_{s t}$ will increase the time of writing in a display data. On the other hand, increasing the channel length of $M_{1}$ is more affordable since the original size of $M_{1}$ is relatively small. For current typical pixel designs, the channel length of $M_{1}$ can be increased by multiple times and its aperture ratio can still be higher than the minimum requirement. Also, increasing the channel length of $M_{1}$ will increase the time of writing in a display data as well. Thus, when increasing the channel length of $M_{1}$, both aperture ratio and the capability of writing in a display data should be maintained higher than their minimum requirement.

\section{Proposed PiXel Design fOR Minimizing DISPLAY-DATA VARIATION}

\section{A. Design Overview and Design Constraints}

In this section, we propose a novel pixel design to minimize the display-data variation resulting from the large leakage current of nc-Si TFTs. Fig. 7 shows the schematic of the proposed pixel design, which consists of three TFTs $\left(M_{3}, M_{4}\right.$, and $\left.M_{5}\right)$, three capacitors $\left(C_{1}, C_{2}\right.$, and $\left.C_{3}\right)$, and one OLED. Compared to the typical design shown in Fig. 4, TFT $M_{5}$ in the proposed pixel design plays the same role as TFT $M_{2}$ in the typical design, which connects a voltage source to the OLED. Instead of using only one capacitor $\left(C_{s t}\right)$ in the typical pixel design, the proposed pixel design uses three capacitors $\left(C_{1}, C_{2}\right.$, and $\left.C_{3}\right)$ to store the display data. Also, the proposed pixel design utilizes two TFTs $\left(M_{3}\right.$ and $\left.M_{4}\right)$ as switches to determines whether the display data can be written in. The voltage at $P_{2}$ determines the lightness of the OLED.

The proposed pixel design can effectively minimize the display-data variation by adding an extra TFT in between the stored display data (voltage at $P_{2}$ ) and Data_line during the datawritten phase. Also, the proposed pixel design requires no extra control signal other than Scan_line and Data_line, and thus can be easily applied to current AM-OLED displays using nc-Si TFTs. However, in order to limit the area overhead and provide an enough aperture ratio, two design constraints need to be followed for the proposed design. First, the total capacitance of $C_{1}, C_{2}$, and $C_{3}$ is the same as $C_{s t}$. Second, the size of each TFT used in the proposed design is the same as that used in 


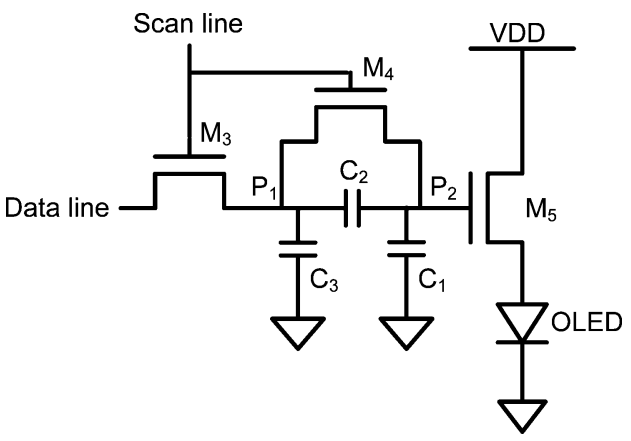

Fig. 7. Schematic of the proposed pixel design.

the typical design. With these two constraints, the area overhead of the proposed pixel design is around one TFT more compared to the typical design, which is within the affordable range based on the aperture ratio of our current pixel design. Actually, this 1-TFT area overhead can be further minimized using proper layout techniques. Note that we strictly limit the size of the total capacitance because the capacitor in the typical pixel design already occupies a big portion of the pixel-cell layout. Any further significant increase of the capacitance may result in large area overhead and in turn an unacceptably low aperture ratio.

In the following Sections IV-B and IV-C, we will first detail the behavior of the proposed pixel design during the data-written phase and the data-retained phase, respectively. Next, we will compare the proposed pixel design with the typical pixel design based on the result of analysis, simulation, and measurement. Also, we will discuss the impact of using different combinations of the sizes of $C_{1}, C_{2}$, and $C_{3}$ on the display-data variation and the data-written time.

\section{B. Circuit Behavior During Data-Written Phase}

During the data-written phase, Scan_line is set to high and both $M_{3}$ and $M_{4}$ are turned on. Once $M_{4}$ is turned on, the voltages at the two terminals of $C_{2}, P_{1}$, and $P_{2}$, are about the same. Then similar data voltage will be written into $C_{3}$ through one TFT $\left(M_{3}\right)$ and be written into $C_{1}$ through two TFTs $\left(M_{3}\right.$ and $M_{4}$ ). As a result, the discharge of $C_{2}$ is relatively small during the data-written phase. Since writing data into $C_{1}$ needs to pass a larger resistance (one more TFT) than writing data into $C_{3}$, a smaller ratio of $C_{1}$ over $C_{s t}$ can reduce the write time of the proposed pixel design.

\section{Circuit Behavior During Data-Retained Phase}

At the beginning of the data-retained phase (right after the data-written phase ends), the voltages at $P_{1}$ and $P_{2}$ should be the same and represent the display data (the impact of the parasitic capacitance of $M_{3}$ and $M_{4}$ is ignored here). At the same time, Scan_line is set to low and both $M_{3}$ and $M_{4}$ are turned off. Due to the leakage of $M_{3}$ and $M_{4}$, the data at $P_{1}$ and $P_{2}$ may be changed. The amount of the leakage current is strongly determined by the TFT's drain-source voltage. As shown in Fig. 6, the leakage current when $V_{d s}=10$ volt could be 3-order larger than the leakage current when $V_{d s}=0.1$ volt. Thus, the leakage of $M_{3}$ can be much larger than that of $M_{4}$ since the voltage between Data_line and $P_{1}$ is significant larger than the voltage between $P_{1}$ and $P_{2}$ (almost 0 at the beginning of the data-retained phase).

Compared to the typical pixel design shown in Fig. 4, the data variation at $P_{1}$ is larger than that at $P_{0}$ since the capacitance of $C_{3}$ is smaller than that of $C_{s t}$. However, the data variation at $P_{2}$ is significantly smaller than that at $P_{1}$ due to a smaller leakage current of $M_{4}$. Note that the voltage at $P_{2}$ represents the display data during the data-retained phase. Thus, the key idea of the proposed design is to protect the data at $P_{2}$ by creating a smaller drain-source voltage of $M_{4}$ while allowing a larger but proper data variation at $P_{1}$. In our later analysis, we will discuss how the setup of the size of $C_{1}, C_{2}$, and $C_{3}$ affects the data variation at $P_{2}$ for the proposed pixel design.

In fact, the voltage between $P_{1}$ and $P_{2}$ will increase with time, and in turn may degrade the capability of the proposed pixel for protecting its display data after a certain period of time. Fortunately, this situation only occurs after the time passes the period of the data-retained phase. Thus, the proposed pixel design can still effectively protect its display data for current applications of display panels.

\section{Worst-Case Analysis for the Proposed PiXel Design}

In this section, we theoretically analyze the degree of display-data variation and the time of writing in a display data for the proposed pixel design, respectively. Also, the analysis for both data variation and write time is performed under their worst case.

\section{A. Worst-Case Display-Data Variation}

The leakage current of a transistor is directly proportional to its drain-source voltage. Thus, the worst case of displaydata variation of a pixel occurs when the display data of the pixel stores the maximum voltage (denoted as $V_{\max }$ ) and its Data_line stays constantly at the minimum voltage (0 volt) during the data-retained phase. Based on this worst-case setting, we then analyze the display-data variation for both the typical and proposed pixel designs, i.e., to calculate the voltage at $P_{0}$ in Fig. 4 and the voltage at $P_{2}$ in Fig. 7 with respective to time $t$, respectively.

1) Display-Data Variation of the Typical Pixel Design: The current flowing in the transistor $M_{1}$ is equal to the current flowing out of the capacitor $C_{s t}$ during its discharging. Thus, the voltage at node $P_{0}$ (denoted as $V_{P_{0}}$ ) can be determined by (3), where $R_{M_{1 \_ \text {of } f}}$ represents the resistance of $M_{1}$ when $M_{1}$ turns off

$$
\frac{V_{P_{0}}}{R_{M_{1} \_f f}}=-C_{s t} \cdot \frac{d V_{P_{0}}}{d t} .
$$

By solving the differential equation of (3), we can obtain $V_{P_{0}}$ versus time as shown in (4). Note that the boundary condition is $V_{P_{0}}=V_{\max }$ when $t=0$

$$
V_{P_{0}}=V_{\max } \cdot e^{\frac{-t}{R_{M_{1}-o f f} \cdot C_{s t}}} .
$$




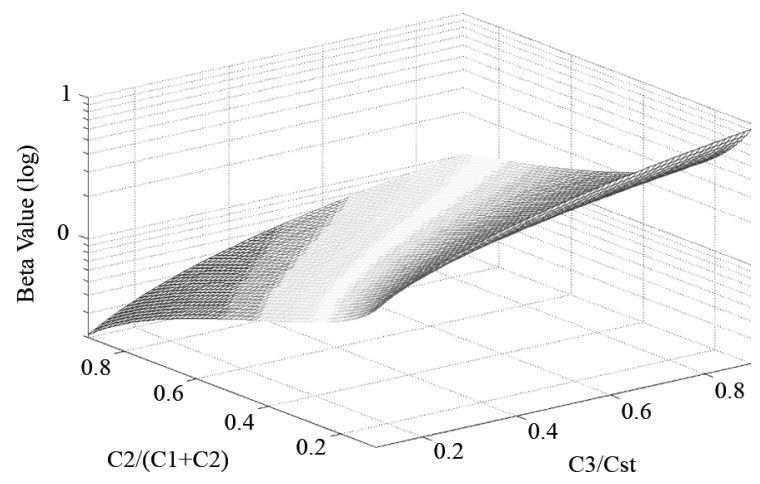

Fig. 8. Beta value associated with different settings of $C_{1}, C_{2}$, and $C_{3}$ when $t=0.01 * R_{\text {off }} * C_{s t}$.

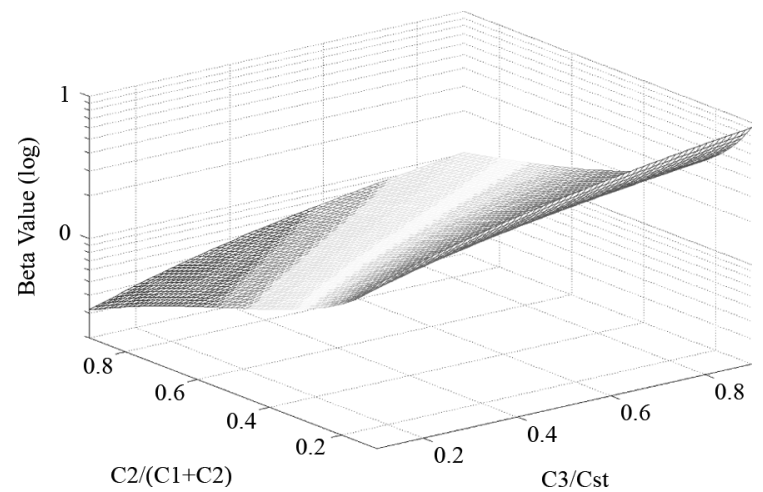

Fig. 9. Beta value associated with different settings of $C_{1}, C_{2}$, and $C_{3}$ when $t=0.1 * R_{\text {off }} * C_{s t}$

Based on (4), we can then derive the derivative of $V_{P_{0}}$ with respect to time $t$ as listed in (5)

$$
\left|\frac{d V_{P_{0}}}{d t}\right|=\left(\frac{V_{\max }}{R_{M_{1} \_f f} \cdot C_{s t}}\right) \cdot e^{\frac{-t}{R_{M_{1}-o f f} \cdot C_{s t}}} .
$$

2) Display-Data Variation of the Proposed Pixel Design: To analyze the voltage at $P_{2}$ (denoted as $V_{P_{2}}$ ) for the proposed pixel design, we need to analyze the voltage at $P_{1}$ (denoted by $V_{P_{1}}$ ) first. During the data-retained phase, the turn-off resistance of $M_{4}$ (denoted as $R_{M_{4-o f f}}$ ) can be viewed as infinite since its $V_{d s}$ is low and the leakage current is much smaller than that of $M_{3}$. As a result, the equivalent capacitance at $P_{1}$ is $\left[C_{3}+\left(C_{2} \| C_{1}\right)\right]$ during the data-retained phase, where $\left(C_{2} \| C_{1}\right)$ represents the equivalent capacitance when $C_{2}$ is connected to $C_{1}$ in serial. Thus, by substituting $C_{s t}$ with $\left[C_{3}+\left(C_{2} \| C_{1}\right)\right]$ and $R_{M_{1 \_ \text {off }}}$ with $R_{M_{3} \text { off } f}$ in (5), we can obtain the derivative of $V_{P_{1}}$ with respect to time $t$ as listed in (6)

$$
\begin{aligned}
\left|\frac{d V_{P_{1}}}{d t}\right|= & \left(\frac{V_{\max }}{R_{M_{3} \_f f} \cdot\left[C_{3}+\left(C_{2} \| C_{1}\right)\right]}\right) \\
& \cdot e^{\frac{-t}{R_{M_{3}-o f f} \cdot\left[C_{3}+\left(C_{2} \| C_{1}\right)\right]}} .
\end{aligned}
$$

Because of the charge sharing between $C_{1}$ and $C_{2}$, the relation between $d V_{P_{1}} / d t$ and $d V_{P_{2}} / d t$ can be obtained as in (7)

$$
\frac{d V_{P_{2}}}{d t} \cong \frac{d V_{P_{1}}}{d t} \cdot\left(\frac{C_{2}}{C_{1}+C_{2}}\right) .
$$

By substituting $d V_{P_{1}} / d t$ with (6), we obtain the derivative of $V_{P_{2}}$ with respect to time as shown in (8)

$$
\begin{aligned}
\left|\frac{d V_{P_{2}}}{d t}\right| \cong & \left(\frac{V_{\max }}{\left.R_{M_{3-o f f} \cdot\left[C_{3}+\left(C_{2} \| C_{1}\right)\right]}\right)}\right. \\
& \cdot e^{\frac{-t}{R_{M_{3}-o f f} \cdot\left[C_{3}+\left(C_{2} \| C_{1}\right)\right]}} \cdot\left(\frac{C_{2}}{C_{1}+C_{2}}\right) .
\end{aligned}
$$

3) Comparison Between the Proposed and Typical Pixel Designs: To evaluate the effectiveness of the proposed pixel deign on reducing the display-data variation, we first define the factor $\beta$ as the ratio of $d V_{P_{0}} / d t$ over $d V_{P_{2}} / d t$. A larger value of $\beta$ means that the proposed pixel design can protect its display data better from the transistor's leakage compared the typical pixel design. Equation (9) shows the calculation of $\beta$ by combining (5) and (8). Note that $R_{\text {off }}=R_{M_{1 \_f f}}=R_{M_{3 \_ \text {off }}}$ since the transistors used in both designs have the same device size

$$
\begin{aligned}
\beta= & \left|\frac{\frac{d V_{P_{0}}}{d t}}{\frac{d V_{P_{2}}}{d t}}\right| \\
\cong & \left(\frac{C_{1}+C_{2}}{C_{2}}\right) \frac{\left[C_{3}+\left(C_{2} \| C_{1}\right)\right]}{C_{s t}} \\
& \cdot e^{\frac{t}{R_{o f f}} \cdot\left\{1 /\left[C_{3}+\left(C_{2} \| C_{1}\right)\right]-1 / C_{s t}\right\}} .
\end{aligned}
$$

Equation (9) shows that the value of $\beta$ is affected by the following factors: 1) the turn-off resistance of the transistor; 2) the size of each capacitor $\left(C_{1}, C_{2}, C_{3}\right.$, and $\left.C_{s t}\right)$; and 3$)$ the time $t$. Figs. 8 and 9 show the logarithm of $\beta$ associated with $C_{3} / C_{s t}$ and $C_{2} /\left(C_{1}+C_{2}\right)$ when $t=0.01 * R_{\text {off }} * C_{s t}$ and $t=0.1 * R_{\text {off }} * C_{s t}$, respectively. From Figs. 8 and 9 , we find the following two trends about the value of $\beta$. Note that $C_{1}+C_{2}+C_{3}=C_{s t}$.

- A larger $C_{3}$ out of the total capacitance $C_{s t}$ leads to a higher $\beta$.

- A smaller $C_{2}$ out of $C_{1}+C_{2}$ leads to a higher $\beta$.

The reason for the first trend is that a larger $C_{3}$ can minimize the voltage variation at $P_{1}$, which in turn can minimize the voltage variation at $P_{2}$ as shown in (7). The reason for the second trend is that a smaller $C_{2} /\left(C_{1}+C_{2}\right)$ can minimize the voltage variation at $P_{2}$ caused by the charge sharing between the serially connected $C_{1}$ and $C_{2}$.

Fig. 10 shows the value of $\beta$ over time for three different settings of $C_{1}, C_{2}$, and $C_{3}$. Note that the turn-off resistance $R_{\text {off }}$ of a TFT varies along with its $V_{d s}$ as shown Fig. 6, where $R_{\text {off }}$ ranges from 10 volt $/ 10^{-10}$ A to 0.1 volt $/ 10^{-13} \mathrm{~A}$, i.e., $10^{11} \Omega$ to $10^{12} \Omega$. With $C_{s t}=200 \mathrm{fF}$, the time represented by $1 *$ $R_{\text {off }} * C_{s t}$ here should be between 20 and $200 \mathrm{~ms}$ (average 110 $\mathrm{ms}$ ). Since the period of the data-retained phase defined in the specification is $16.7 \mathrm{~ms}$ (corresponding to $60 \mathrm{~Hz}$ ), we care only the value of $\beta$ before $0.2 * R_{\text {off }} * C_{s t}$. During this period ( 0 to $0.2 * R_{\text {off }} * C_{s t}$ ) in Fig. 10, the value of $\beta$ is almost a constant for all capacitance settings.

However, the above analysis is only an approximation since the TFT transistor $M_{4}$ is assumed to be fully turned off during the data-retained phase. In reality, a smaller $C_{2}$ may not always lead to a higher $\beta$ because a larger $C_{2}$ can help to reduce the variation of $V_{d s}$ of $M_{4}$ and in turn limit the leakage current of $M_{4}$. Fig. 11 shows the $V_{d s}$ of $M_{4}$ over time with different values of 


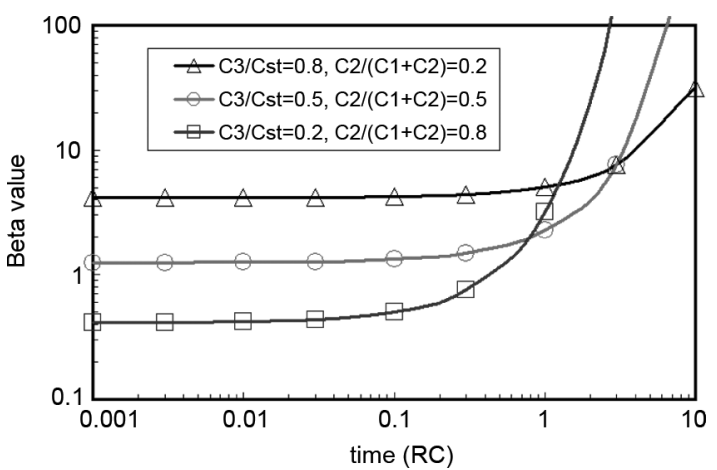

Fig. 10. $\beta$ over time for three capacitance settings.

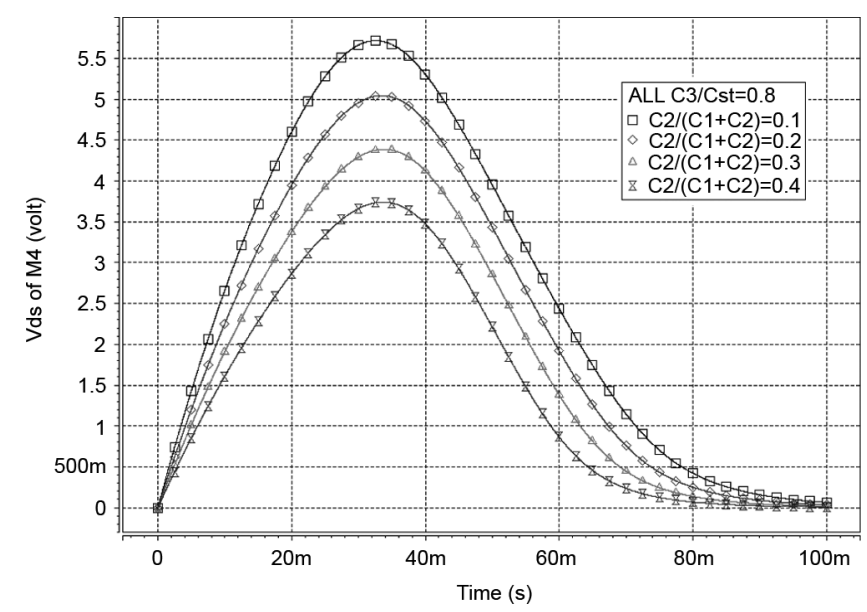

Fig. 11. $V_{d s}$ of $M_{4}$ over time associated with different values of $C_{2}$ given $C_{3} / C_{s t}=0.8$.

$C_{2}$, where the $V_{d s}$ of $M_{4}$ increases while $C_{2}$ decreases. If a too small $C_{2}$ is used, the leakage of $M_{4}$ will significantly increase, which will then speed up the voltage variation at $P_{2}$. Thus, the size of $C_{2}$ should be controlled within a proper range to minimize the display-data variation. This constraint also implies that the size of $C_{3}$ cannot be too large since the total capacitance of $C_{1}, C_{2}$, and $C_{3}$ is a constant $\left(C_{s t}\right)$. A too large $C_{3}$ will limit the size of $C_{2}$.

Since the $V_{d s}$ of $M_{4}$ is originally 0 at the beginning and then gradually becomes larger, the leakage of $M_{4}$ becomes larger along with the time, meaning that the proposed pixel design can only protect its display data effectively at the early stage and may gradually lose its capability of data protection later on. Fortunately, the increase of the $V_{d s}$ of $M_{4}$ in our proposed pixel design is not significant enough during the data-retained phase defined in our specification $(16.7 \mathrm{~ms}$, reciprocal of $60 \mathrm{~Hz})$. Thus, compared to the typical design, the proposed pixel design can still effectively protect the display data with a proper setting of its three capacitors. We will show the simulation result to validate this phenomenon in the next section.

\section{B. Worst-Case Write Time}

In this subsection, we analyze the worst-case write time, i.e., writing a $V_{\max }$ into a pixel whose original value is 0 volt, for both typical and proposed pixel designs. The write time of a pixel design is determined by its equivalent $R C$ time constant.

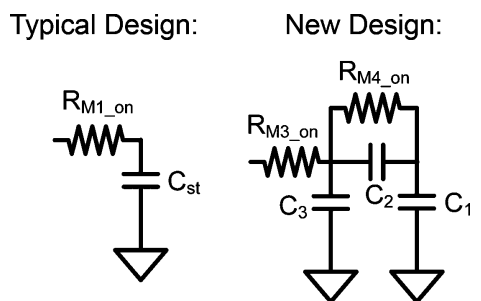

Fig. 12. Illustration of the $R C$ model during the data-written phase for both the typical and proposed designs.

Fig. 12 illustrates the simplified $R C$ model for both the typical and the proposed new pixel design, where $R_{M_{x-} \text { on }}$ represent the resistance of the transistor $M_{x}$ when $M_{x}$ turns on.

Based on the $R C$ model of Fig. 12, (10) and (11) shows the worst-case write time for the typical design (denoted as $W T_{\text {typ }}$ ) and the proposed design (denoted as $W T_{\text {pro1 }}$ ), respectively. Note that the transistors on both designs have the same device size and hence $R_{\mathrm{on}}=R_{M_{1-o n}}=R_{M_{3-o n}}=R_{M_{4-o n}}$

$$
\begin{aligned}
W T_{\mathrm{typ}} \propto & R_{M_{1-o n}} \cdot C_{s t}=R_{\mathrm{on}} \cdot C_{s t} \\
W T_{\mathrm{pro1}} \propto & \left(R_{M_{3 \_} \text {on }}+R_{M_{4-o n}}\right) \cdot C_{1} \\
& +R_{M_{4-o n}} \cdot C_{2}+R_{M_{3-o n}} \cdot C_{3} \\
= & R_{o n} \cdot\left(2 C_{1}+C_{2}+C_{3}\right) \\
= & R_{o n} \cdot\left(C_{s t}+C_{1}\right) .
\end{aligned}
$$

In fact, the $W T_{\text {prol }}$ in (11) represents the upper bound of the worst-case write time of the proposed design. The term $R_{M_{4}-o n}$. $C_{2}$ is overestimated since the voltage between $P_{1}$ and $P_{2}$ is small during the data-written phase (small $R_{M_{4-o n}}$ ) and $C_{2}$ is not fully discharged. If we completely ignore the impact of $C_{2}$ and eliminate the term $R_{M_{4-} \text { on }} \cdot C_{2}$ from (11), a lower bound of the worst-case write time can be obtained as in (12)

$$
\begin{aligned}
W T_{\mathrm{pro} 2} & \propto\left(R_{M_{3} \text { on }}+R_{M_{4-o n}}\right) \cdot C_{1}+R_{M_{3-o n}} \cdot C_{3} \\
& =R_{\mathrm{on}} \cdot\left(C_{s t}+C_{1}-C_{2}\right) .
\end{aligned}
$$

As (11) shows, the upper bound of the worst-case write time of the proposed design is $R_{\mathrm{on}} C_{1}$ more than that of the typical design. As (12) shows, the lower-bound of the worst-case write time is $R_{\mathrm{on}} C_{2}$ less than the upper bound. Thus, to reduce the write time for the proposed design, $C_{1}$ needs to be smaller and $C_{2}$ needs to be larger. However, a smaller $C_{1}$ and a larger $C_{2}$ may decrease pixel's capability of protecting the display data. In Section VI, we discuss how to find a proper setting of all capacitors used in the proposed pixel design through simulation.

\section{Simulation Results}

In this section, we will discuss the following issues through the simulation results, which are performed based on the PECVD-based bottom-gate nc-Si-TFT technology developed by ITRI [8].

\section{A. Worst-Case Display-Data Variation}

In the following experiment, we simulate the display-data variation of the proposed design by using different settings of its capacitors $\left(C_{1}, C_{2}\right.$, and $\left.C_{3}\right)$, and then compare it to that of the typical design. The display-data variation is defined by the 


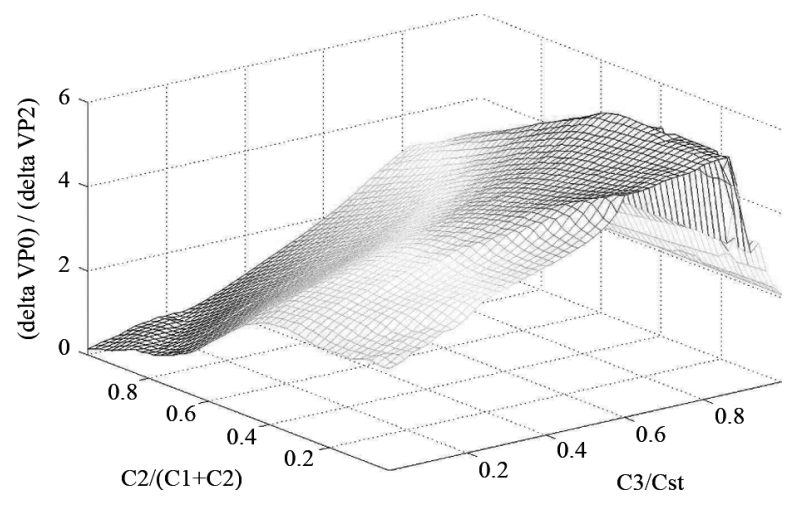

Fig. 13. $\Delta V_{P_{0}} / \Delta V_{P_{2}}$ associated with different capacitance settings.

biggest voltage difference to its original stored voltage (at $P_{2}$ or $P_{0}$ ) caused during a whole data-retained phase. We use $\Delta V_{P_{0}}$ and $\Delta V_{P_{2}}$ to denote this display-data variation for the typical and the proposed designs, respectively. The stored display data here is set to $V_{\max }$, i.e., 10 volt in our specification, and its Data_line is enforced to stay at 0 volt such that the leakage current of the pixel can be the worst. The total capacitor in use $\left(C_{s t}\right)$ is $200 \mathrm{fF}$. The channel length and width of a TFT transistor are both $8 \mu \mathrm{m}$. The period of a data-retained phase is 16.7 ms (reciprocal of $60 \mathrm{~Hz}$ ). Fig. 13 shows the ratio of $\Delta V_{P_{0}}$ over $\Delta V_{P_{2}}$ for different combination of $C_{3} / C_{s t}$ and $C_{2} /\left(C_{1}+C_{2}\right)$, where $C_{1}+C_{2}+C_{3}=C_{s t}$.

As the result shows, Fig. 13 confirms our inferences about display-data variation in Section V-A. First, $\Delta V_{P_{0}} / \Delta V_{P_{2}}$ increases when $C_{3}$ increases initially but then starts to drop after $C_{3}$ is larger than a certain value. Second, $\Delta V_{P_{0}} / \Delta V_{P_{2}}$ also increases when $C_{2}$ decreases initially but then starts to drop after $C_{2}$ is smaller than a certain value. The peak of $\Delta V_{P_{0}} / \Delta V_{P_{2}}$ occurs when $C_{3} / C_{s t}=0.86$ and $C_{2} /\left(C_{1}+C_{2}\right)=0.32$. Also, the peak value of $\Delta V_{P_{0}} / \Delta V_{P_{2}}$ is more than 5.65, meaning that the proposed design can effectively reduce the data variation by 5.65 times compared to the typical design.

Next, Fig. 14 shows the $V_{P_{0}}$ and $V_{P_{2}}$ versus time, given that $C_{s t}=200 \mathrm{fF}, C_{3} / C_{s t}=0.86$, and $C_{2} /\left(C_{1}+C_{2}\right)=0.32$. The rest experiment setting is the same as that used for Fig. 13. As the result shows, the display data of the proposed design $\left(V_{P_{2}}\right)$ drops much slower than the typical design at the beginning but then drops faster after a certain period of time. In this experiment, $V_{P_{2}}$ starts to drop faster than $V_{P_{0}}$ when $t$ is larger than $37.5 \mathrm{~ms}$, which is much longer than the normal specification of the data-retained phase, $16.7 \mathrm{~ms}$. This result further demonstrates the effectiveness of the proposed pixel design on reducing the display-data variation for practical applications of display panels.

\section{B. Worst-Case Write Time}

Fig. 15 shows the ratio of the worst-case write time of the proposed design (denoted as $W T_{\text {pro }}$ ) over that of the typical design (denoted as $W T_{\text {typ }}$ ) for different capacitance settings. As the result shows, the write time of the proposed design decrease when $C_{2}$ increases or $C_{1}$ decreases, which fits the conclusion of our analysis in Section V-B.

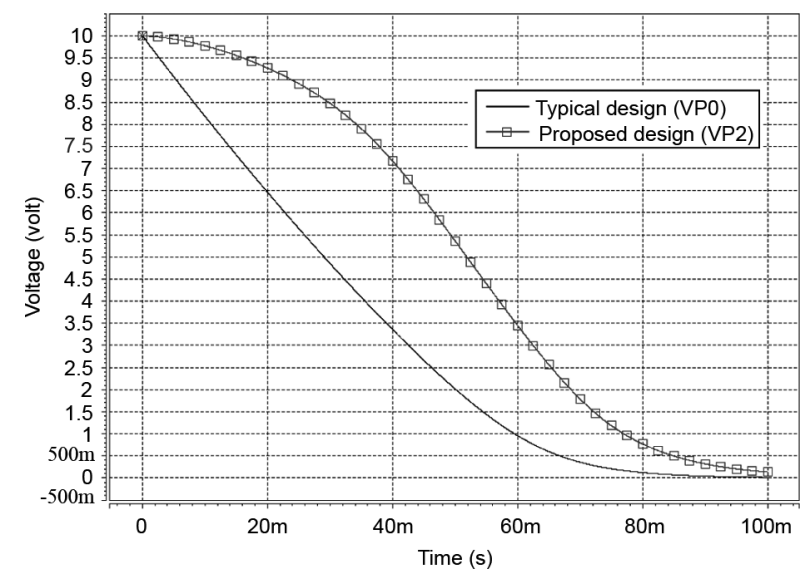

Fig. 14. Stored display data over time for both the typical and proposed designs.

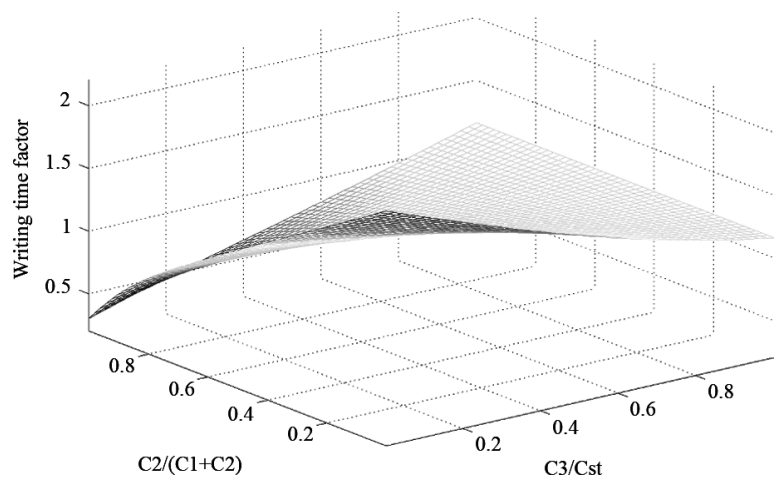

Fig. 15. $W T_{\text {pro }} / W T_{\text {typ }}$ associated with different capacitance settings.

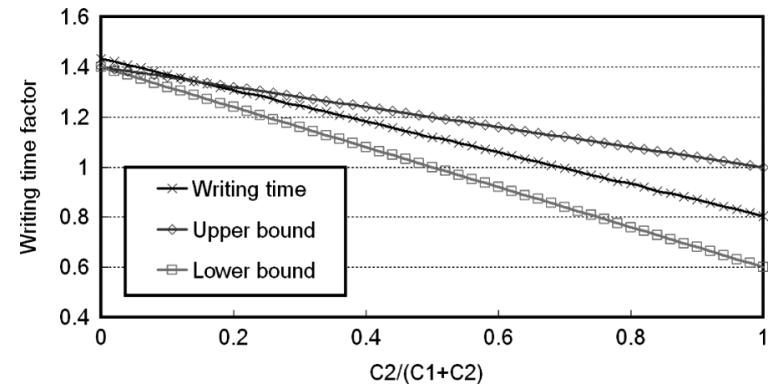

Fig. 16. $W T_{\text {pro }} / W T_{\text {typ }}$ versus $C_{2} /\left(C_{1}+C_{2}\right)$ when $C_{3} / C_{s t}=0.6$.

Fig. 16 further shows the $W T_{\text {pro }} / W T_{\text {typ }}$ obtained by the simulation, the upper bound in (11), and the lower bound in (12) when $C_{3} / C_{s t}$ is set to 0.6 and $C_{2} /\left(C_{1}+C_{2}\right)$ varies. The result again confirms our analysis on the write time for the proposed design.

\section{Finding a Proper Capacitance Setting}

In general, the write time of the pixel design is usually a constraint instead of a factor to be minimized. Thus, in this subsection, we attempt to find a capacitance setting for the proposed design which can result in the largest $\Delta V_{P_{0}} / \Delta V_{P_{2}}$ under the constraint that its $W T_{\text {pro }} / W T_{\text {typ }}$ is not more than 1.15 . We first find the capacitance setting with the largest $\Delta V_{P_{0}} / \Delta V_{P_{2}}$, 


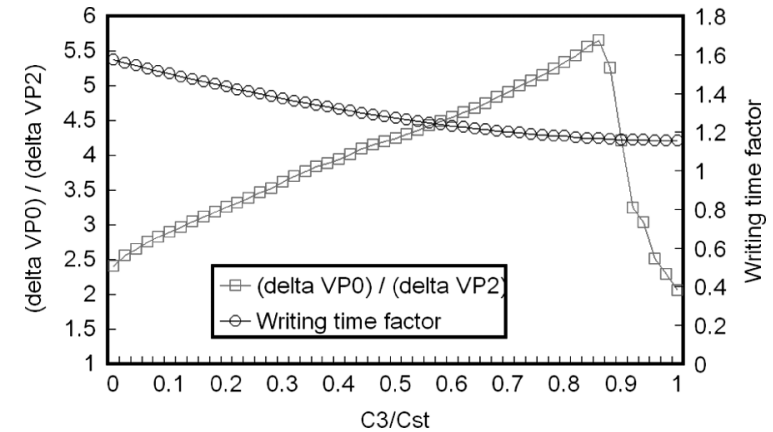

Fig. 17. $\Delta V_{P_{0}} / \Delta V_{P_{2}}$ and $W T_{\text {pro }} / W T_{\text {typ }}$ versus $C_{3} / C_{s t}$ when $C_{2} /\left(C_{1}+C_{2}\right)=0.32$.

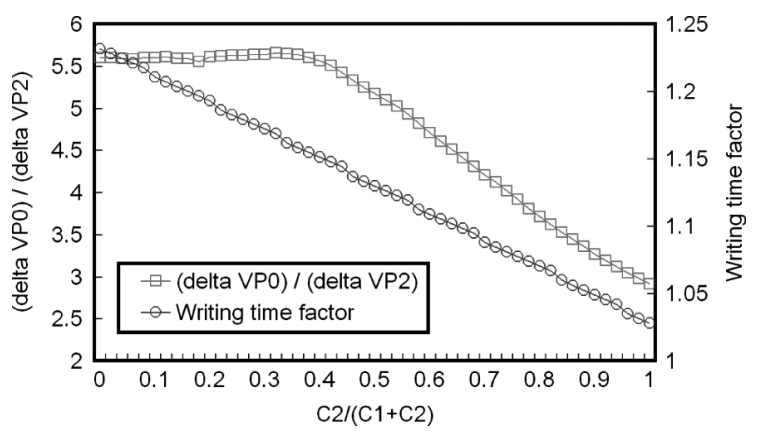

Fig. 18. $\Delta V_{P_{0}} / \Delta V_{P_{2}}$ and $W T_{\text {pro }} / W T_{\text {typ }}$ versus $C_{2} /\left(C_{1}+C_{2}\right)$ when $C_{3} / C_{s t}=0.86$.

i.e., $C_{3} / C_{s t}=0.86$ and $C_{2} /\left(C_{1}+C_{2}\right)=0.32$ as shown in Fig. 13. For $C_{3} / C_{s t}=0.86$ and $C_{2} /\left(C_{1}+C_{2}\right)=0.32$, its $\Delta V_{P_{0}} / \Delta V_{P_{2}}$ is 5.65 but its $W T_{\text {pro }} / W T_{\text {typ }}$ is 1.17 , which exceeds the constraint of 1.15 . We then search around the capacitance setting around the peak point, $C_{3} / C_{s t}=0.86$ and $C_{2} /\left(C_{1}+C_{2}\right)=0.32$. Fig. 17 shows both $\Delta V_{P_{0}} / \Delta V_{P_{2}}$ and $W T_{\text {pro }} / W T_{\text {typ }}$ when $C_{2} /\left(C_{1}+C_{2}\right)$ is fixed at 0.32 and $C_{3} / C_{s t}$ varies. As the result shows, the improvement of the proposed design on the display-data variation drops rapidly when $C_{3} / C_{s t}$ is over 0.86 but the corresponding write time does not drop significantly. Thus, $C_{3} / C_{s t}=0.86$ appears to be the best setting.

Next, Fig. 18 shows $\Delta V_{P_{0}} / \Delta V_{P_{2}}$ and $W T_{\text {pro }} / W T_{\text {typ }}$ when $C_{3} / C_{s t}$ is set to 0.86 but $C_{2} /\left(C_{1}+C_{2}\right)$ varies. As the result shows, the improvement on the display-data variation stays around the same level when $C_{2} /\left(C_{1}+C_{2}\right)$ is near the peak point 0.32 . Thus, we can then choose the capacitance setting in Fig. 18 which results in the largest $\Delta V_{P_{0}} / \Delta V_{P_{2}}$ with $W T_{\text {pro }} / W T_{\text {typ }}<1.15$, i.e., $C_{2} /\left(C_{1}+C_{2}\right)=0.4$ and $C_{3} / C_{s t}=0.86$. Its improvement on display-data variation can be $5.55 \times$ and its $W T_{\text {pro }} / W T_{\text {typ }}$ is exactly 1.15 .

Note that designers may need to consider more design parameters other than the write time and the display-data variation, such as layout area or aperture ratio. The capacitance setting for the proposed design should be chosen based on the corresponding objective and design constraints in use. In fact, several capacitance settings in Fig. 13 can achieve a $\Delta V_{P_{0}} / \Delta V_{P_{2}}$ close to the maximum. The designers could utilize those settings for optimizing other design parameters.

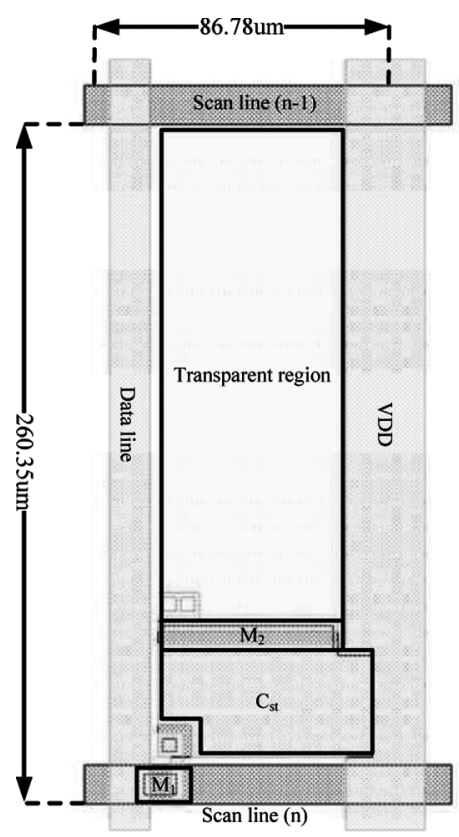

(a)

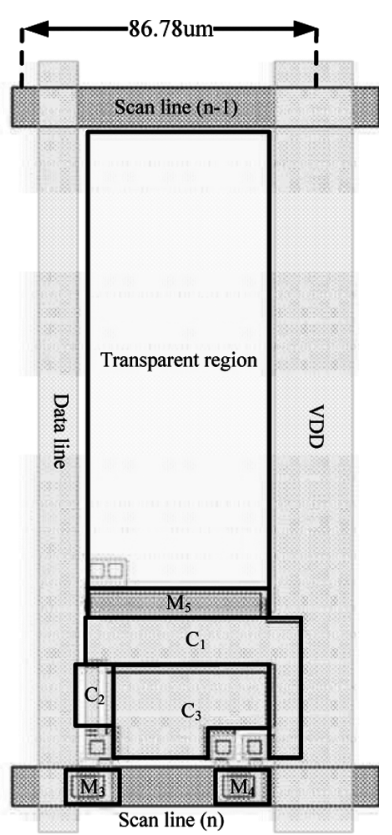

(b)
Fig. 19. Layout of the typical and proposed designs for a 4.1" QVGA color AM-OLED panel. (a) Typical design. (b) Proposed design.

\section{Area Overhead and Aperture Ratio}

Fig. 19(a) and (b) illustrate the layout of the typical pixel design and the the proposed pixel design, respectively. Both pixel designs follows the specification of 4.1" QVGA color AM-OLED backplanes. Its resolution is $320 \times R G B \times 240$ and its pixel size is $86.78 \mu \mathrm{m} \times 260.35 \mu \mathrm{m}$ for each of the red, green, and blue pixels. For our application, we utilize a white-light OLED along with a corresponding color filter to generate each of RGB colors instead of using an individual OLED which can directly generate the corresponding color. Thus, the pixel sizes as well as their required aperture ratios are the same for all red, green, and blue pixels.

Compared to the typical design, the area overhead of the proposed pixel design is an extra transistor $\left(M_{4}\right)$. The total capacitances for both designs are the same. However, as shown in the bottom-right of Fig. 19(b), this transistor $\left(M_{4}\right)$ can be placed above the metal of Scan_line, and hence its resulting area overhead for the control design can be reduced, which in turn limits the decrease of the aperture ratio for the proposed design. Overall, the aperture ratios of the the proposed design is $41.4 \%$, which is $3.2 \%$ lower than that of the typical design $(44.6 \%)$ and is still higher than the minimum requirement of the aperture ratio (40\%) for most AM-OLED displays. This 3.2\% lose on the aperture ratio results from the extra contact holes and the spacing around the two more capacitors enforced by the design rules.

Note that, for both typical and proposed designs, the area occupied by the capacitors is much more than that occupied by the transistors. That is why we are only willing to spend extra area for transistors to reduce the display-data variation, but not extra area for capacitors. The same percent increase in the total capacitance can result in a significant lose in its aperture ratio. 


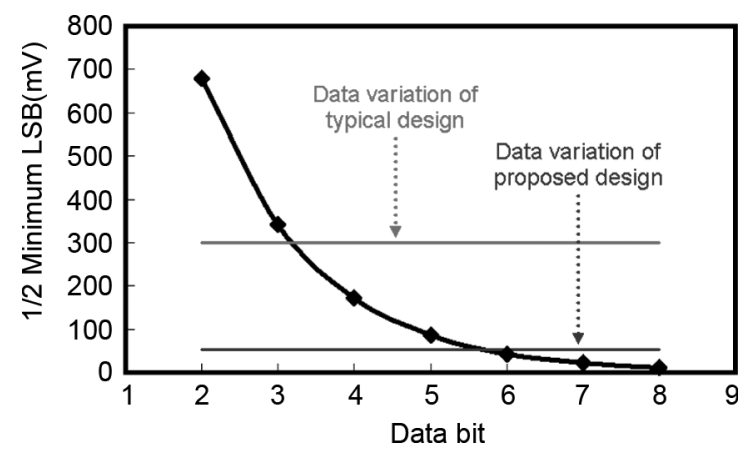

Fig. 20. Improvement of the contrast resolution achieved by using the proposed pixel.

In addition, for the AM-OLED applications which require a higher requirement of the aperture ratio, we can further eliminate the $V_{D D}$ metal line with other signal metal lines [13]. Also, top-emission scheme can be used to provide a higher aperture ratio [14], [15].

\section{E. Improvement on Contrast Resolution}

In this subsection, we attempt to identify the contrast resolution which can be achieved by our current nc-Si-TFT technology [8] with and without applying the proposed pixel design. The supportable contrast resolution for a nc-Si-TFT technology is determined by its display-data variation during the data-retained phase and the voltage of its LSB (least-significant-bit) after $\Gamma$-correction. As long as the corresponding display-data variation is less than one half of the LSB voltage, this contrast resolution is supportable.

Fig. 20 shows one half of the LSB voltage required by different numbers of resolution bits. Also, Fig. 20 shows the display-data variation caused by the typical design and the proposed design, respectively. As the result shows, the typical design can only support a 3-bit contrast resolution with our current nc-Si-TFT technology while the proposed design can support a 5-bit contrast resolution. For general display applications, the number of resolution bits is 8 .

\section{OTHER ISSUES FOR THE PROPOSED PIXEL DESIGN}

\section{A. Turn-Off Voltage at Scan Line}

As Fig. 6 shows, $I_{d s}$ does not always decrease when $V_{g s}$ decreases, meaning that decreasing the turn-off voltage at Scan_line may not be always able to decrease the leakage current. Also, the $V_{g s}$ leading to the minimum $I_{d s}$ also varies with different $V_{d s}$. Thus, in this subsection, we attempt to find an appropriate turn-off voltage at Scan_line (denoted as $V_{S L}$ ) such that the display-data variation for a pixel can be minimized. We first denote the $V_{g s}$ leading to the minimum $I_{d s}$ given $V_{d s}=x$ as $\left.M L_{-} V_{g s}\right|_{V_{d s}=x}$. In Fig. 6, $\left.M L_{-} V_{g s}\right|_{V_{d s}=10 \mathrm{volt}}$ is -6.41 volt and $\left.M L_{-} V_{g s}\right|_{V_{d s}=0.1 \text { volt }}$ is -8.48 volt, where $V_{\max }=10$ volt and $V_{\min }=0$ volt.

The ideal case for a good setting of $V_{S L}$ is that its resulting $V_{g s}$ can be close to $\left.M L_{-} V_{g s}\right|_{V_{d s}}$ for each $V_{d s}$. However, $V_{d s}$ is determined by the voltage at Data_line, which may vary randomly and hard to predict. Also, $V_{d s}$ is determined by the

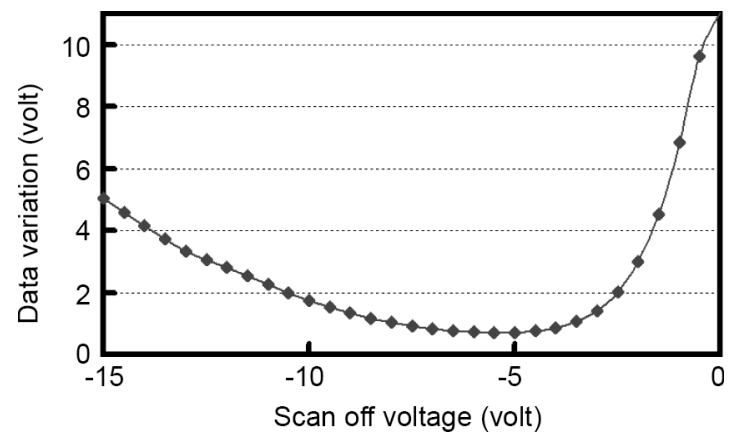

Fig. 21. Worst-case display-data variation versus $V_{S L}$.

voltage of the stored data, which further determines the corresponding $\left.M L_{-} V_{g s}\right|_{V_{d s}}$. It makes the analysis for the best $V_{S L}$ even harder. Thus, we can only know that the best $V_{S L}$ should be around $\left.M L_{-} V_{g s}\right|_{V_{d s}=V_{\max }}$ since the scale of $I_{d s}$ when $V_{d s}=$ $V_{\max }$ is several orders larger than that when $V_{d s}=V_{\min }$.

Fig. 21 shows the display-data variation with different settings of $V_{S L}$, where the store data is set as $V_{\max }$ to create a worst case and the voltage at Data_line is a full-range triangle wave to approximate the random data in real applications. As the result shows, the display-data variation can be minimized when setting the turn-off voltage at Scan_line to -5.5 volt, which is $\left.M L_{-} V_{g s}\right|_{V_{d s}=10 \text { volt }}(-6.41$ volt $)$ plus a small constant.

\section{B. Coupling Effect}

The coupling effect of a pixel design is referred to the transient voltage drop of the stored data caused by the signal transition at Scan_line (from 1 to 0 ) interacting with the parasitic capacitors of switch transistors. As a result, at the beginning of the data-retained phase, the stored value first drops by the coupling effect and then varies by switch transistor's leakage (discussed as data variation previously). This coupling effect has been existed on the pixel designs of AM-LCD and AM-OLED displays. Fig. 22(a) illustrates the coupling effect on the typical pixel design of AM-OLED displays. Fortunately, on the typical pixel design, the voltage drop caused by the coupling effect is almost a constant value for different stored data voltages. It means that by elevating every stored data voltage with a fixed constant, the displayed lightness can remain almost the same as if no coupling effect exists. This solution to the coupling effect can be implemented easily without significant overhead [16] and has already been used for AM-LCD displays.

For the proposed pixel design, the coupling effect becomes larger since the impact of two more parasitic capacitors $\left(C_{g d}\right.$ and $C_{g s}$ ) of $M_{4}$ is taken into account other than the $C_{g s}$ of $M_{3}$ [as shown in Fig. 22(b)]. Fig. 23 shows the voltage drop caused by the coupling effect corresponding to each stored data voltage on the typical design (denoted by Typ) and the proposed designs with 3 arbitrary capacitance settings (denoted by $P_{1}$, $P_{2}$, and $\left.P_{3}\right)$. The capacitance settings are $(P 1) C_{3} / C_{s t}=0.4$, $C_{2} /\left(C_{1}+C_{2}\right)=0.3 ;(P 2) C_{3} / C_{s t}=0.5, C_{2} /\left(C_{1}+C_{2}\right)=$ 0.2 ; and $(P 3) C_{3} / C_{s t}=0.6, C_{2} /\left(C_{1}+C_{2}\right)=0.1$. As the result shows, the voltage drop of the proposed design is around the same for each stored data voltage just like the typical design, and the same trend can be observed on different capacitance 

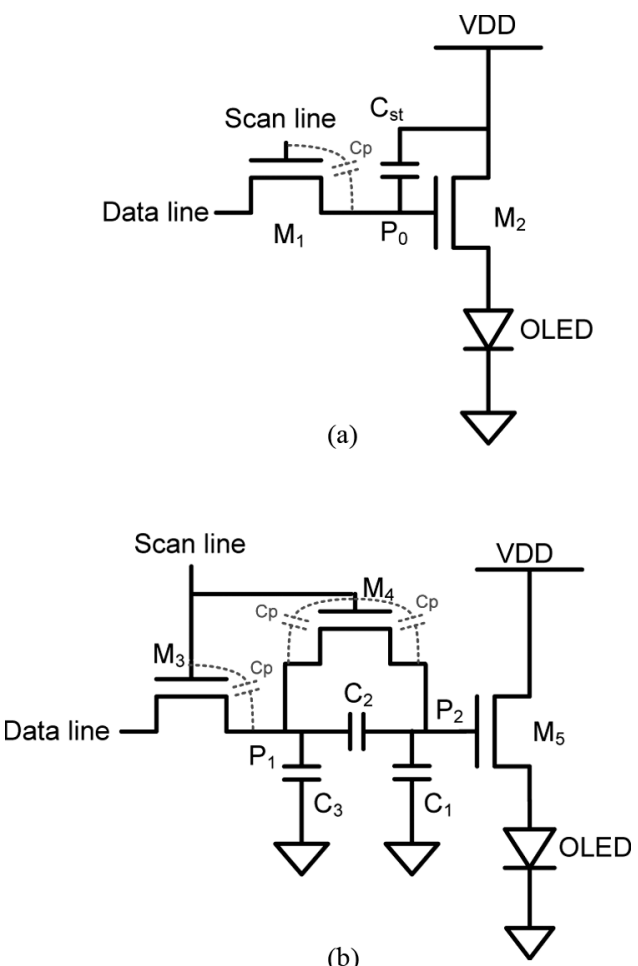

Fig. 22. Parasitic capacitance of the switch TFTs for both the typical and the proposed pixel design. (a) Typical design. (b) Proposed design.

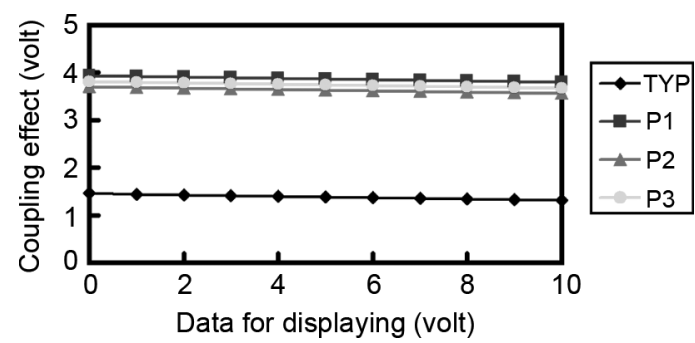

Fig. 23. Coupling effect associated with different stored data for the typical and proposed designs.

settings of the proposed design. It means that the coupling effect can also be solved for the proposed design with the same simple solution described above.

\section{MeAsurement of FABRicated TFT Circuits}

\section{A. Measurement Procedure}

We have fabricated both the typical and the proposed pixel design using the PECVD-based bottom-gate nc-Si-TFT technology developed by ITRI [8]. Fig. 24 shows the photo of a fabricated die, which contains several single pixel designs with different configurations. If we directly probe the voltage of the stored data $\left(V_{P_{0}}\right.$ or $\left.V_{P_{2}}\right)$, the prober of the oscilloscope will impose a $10 \mathrm{pF}$ capacitance and a $1 \mathrm{M} \Omega$ resistance to the pixel design. Thus, we need to measure the voltage at the source of the transistor driving the OLED through an IO pad and then infer the voltage of the corresponding stored data. Fig. 25 illustrates the measurement setting for both the typical and the proposed designs. Note that the fabricated pixel designs are not connected

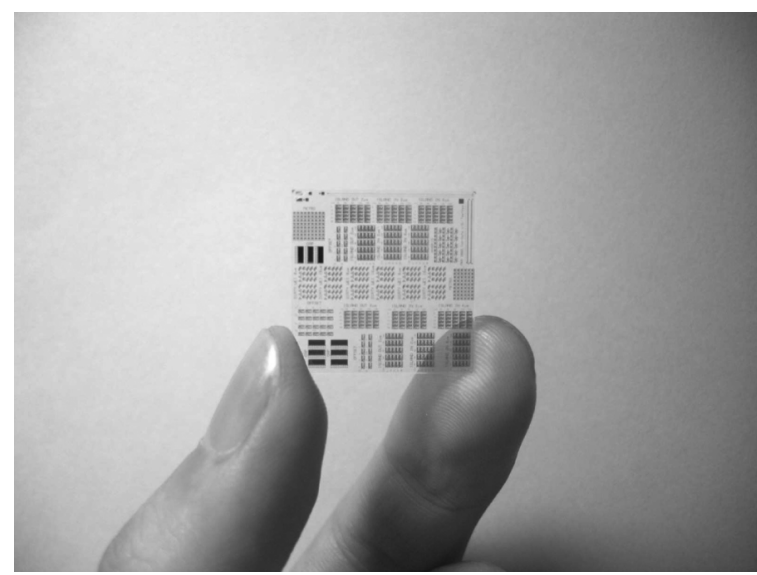

Fig. 24. Photo of the fabricated die.

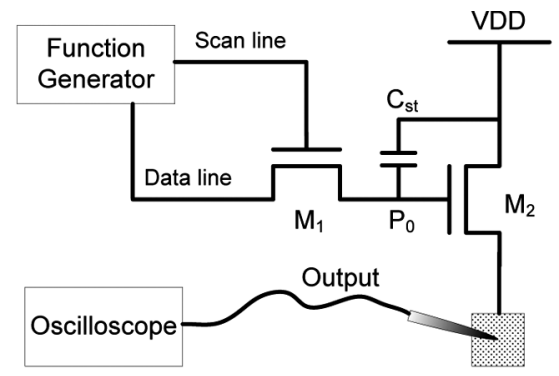

(a)

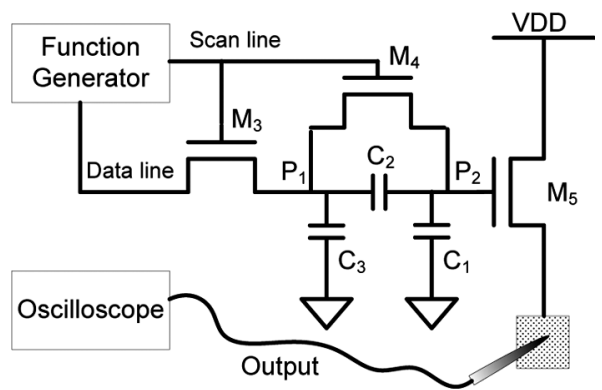

(b)

Fig. 25. Measurement setting for both the typical and proposed designs (a) Typical design. (b) Proposed design

to any OLED. The OLED is replaced by an IO pad for measurement use.

In order to infer the stored data of a pixel $\left(V_{P_{0}}\right.$ or $\left.V_{P_{2}}\right)$, we need to build the relation between the voltage at the gate (denoted as $V_{g}$ ) and the voltage at the source (denoted as $V_{s}$ ) of the TFT transistor driving the OLED (i.e., $M_{2}$ or $M_{5}$ ). For the fabricated typical and proposed designs, the device dimension of both $M_{2}$ and $M_{5}$ is $40 \mu \mathrm{m} / 8 \mu \mathrm{m}$. Fig. 26(a) illustrates how we built the relation between $V_{g}$ and $V_{s}$ of $M_{2}$ (or $M_{5}$ ), where we generate a $1 \mathrm{~Hz}$ triangle wave ranging from 0 volt to 10 volt at $V_{g}$ and then measure the corresponding $V_{s}$ through an IO pad. The transistor's drain is connected to $V_{D D}$ (10 volt). Fig. 26(b) shows the measured $V_{s}$ versus $V_{g}$. Due to the noise of the measured result, we utilize a monotonically-increasing sixth-order polynomial to fit this curve and use the polynomial to map the measured $V_{s}$ to $V_{g}$. Note that this relation between $V_{g}$ and $V_{s}$ is characterized combining the effect of the prober's parasitic 


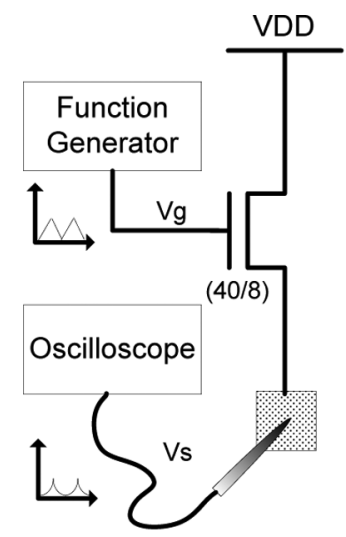

(a)

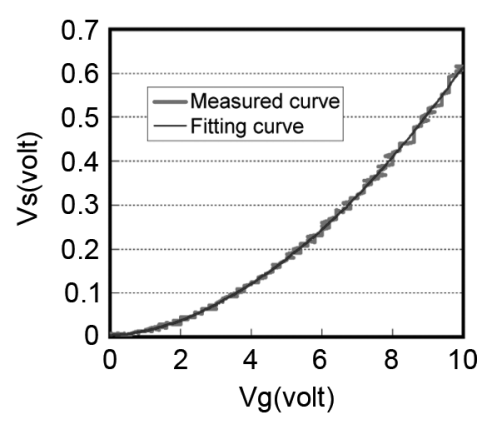

(b)
Fig. 26. $V_{s}$ versus $V_{g}$ of the TFT driving the OLED.

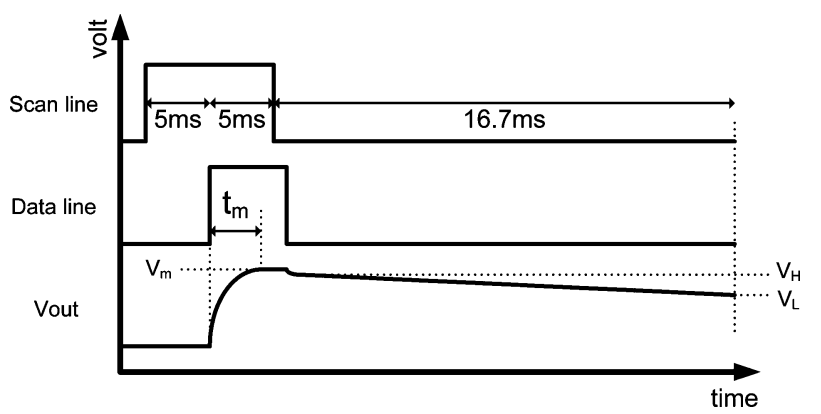

Fig. 27. Signal at Scan_line and Data_line and an exemplary measured result.

capacitance and resistance, which is exactly the same condition when measuring the fabricated pixel designs. Thus, no compensation needs to be added into this characterized $V_{g}-V_{s}$ relation when it is used to infer the stored data of a pixel design.

Fig. 27 shows the signal at Scan_line and Data_line during our measurement as well as an exemplary measured voltage at the output IO pad (denoted as $V_{\text {out }}$ ). When Scan_line just turns on, Data_line keeps on 0 volt for $5 \mathrm{~ms}$ to make sure that the stored data is set to 0 volt. Next, Data_line is set to 10 volt and we start to measure the write time. After another $5 \mathrm{~ms}$, we turns off Scan_line and start to measure the display-data variation during the next $16.7 \mathrm{~ms}$ (the period of the data-retained phase). Note that, due to the coupling effect mentioned previously, the voltage setting of the Data_line has been slightly adjusted for equal stored data in both the typical and the proposed design.

To obtain the display-data variation, we measure the highest $V_{\text {out }}$ (denoted as $V_{H}$ ) and the lowest $V_{\text {out }}$ (denoted as $V_{L}$ ) during the $16.7 \mathrm{~ms}$ data-retained phase. Then we map $V_{H}$ and $V_{L}$ into their corresponding $V_{P_{2}}$ (or $V_{P_{0}}$ ) using the characterized $V_{g}-V_{s}$ relation shown in Fig. 26(b). The difference between the two mapped $V_{P_{2}}$ is the measured display-data variation.

To obtain the write time, we measure the time from $V_{\text {out }}=0$ to $V_{\text {out }}=V_{m}$ (denoted as $t_{m}$ ), where $V_{m}$ is the maximum voltage when Scan_line is on. The measured write time is equal to $t_{m}$ minus the gate-source delay of the transistor. Fig. 28 shows how we obtain the gate-source delay, where a square wave is generated at the gate and measure the time when $V_{s}$ reach the top. In fact, we only choose to use the time when $V_{s}$

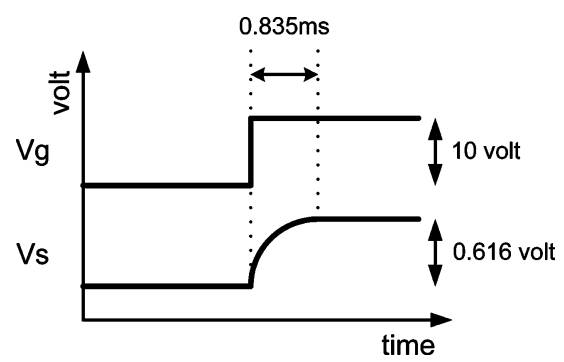

Fig. 28. Measurement of the gate-source delay.

TABLE II

MEASUREMENT RESUlTS FOR 3 DIFFERENT CONFIGURATIONS OF THE TYPICAL PIXEL DESIGN

\begin{tabular}{|c|c|c|c|c|}
\hline \multicolumn{5}{|c|}{ Typical design } \\
\hline Config. & $\begin{array}{c}(\mathrm{W} / \mathrm{L}) \\
\text { of } M_{1}\end{array}$ & $C_{\text {st }}$ & $\begin{array}{c}\text { data var. } \\
\text { ratio }\end{array}$ & $\begin{array}{c}\text { write time } \\
\text { ratio }\end{array}$ \\
\hline T-1 & $(8 / 8)$ & \multirow{3}{*}{$200 f F$} & 1 & 1 \\
\hline T-2 & $(8 / 16)$ & 1.22 & 1.98 \\
\hline T-3 & $(8 / 24)$ & & 2.38 & 3.40 \\
\cline { 5 - 5 } \cline { 4 - 5 } & &
\end{tabular}

reaches the $98 \%$ of the top as the gate-source delay since the signal above the $98 \%$ vibrates severely and it takes long time to reach the true top of $V_{s}$. Similarly, when measuring $t_{m}$, we also choose the time when the measured $V_{\text {out }}$ reaches the $98 \%$ of $V_{m}$.

\section{B. Measurement Result}

Table II lists the measured display-data variation and write time for three configurations of the fabricated typical pixel designs, denoted as T-1, T-2, and T-3. The difference among the three configurations is the channel length of their $M_{1}$ TFT transistor, where the channel length of T-2 and T-3 is double and triple of that of T-1, respectively. The reported display-data variation for a configuration $\mathrm{T}-\mathrm{x}$ is actually a ratio compared to that of T-1, which is $\Delta V_{P_{0}}$ of T-1 over $\Delta V_{P_{0}}$ of $\mathrm{T}-\mathrm{x}$. The reported write time for a configuration $\mathrm{T}-\mathrm{x}$ is the write time of $\mathrm{T}-\mathrm{x}$ over that of $\mathrm{T}-1$.

As the result shows, increasing the channel length of $M_{1}$ can indeed reduce the display-data variation. However, its writetime overhead is directly proportional to its channel length. If we triple the channel length, its measured write time will increase more than 3 times. Also, its improvement on display-data variation is only 2.38 . This result further demonstrates that increasing the channel length of $M_{1}$ is not a cost-effective method to reduce the display-data variation.

Table III lists the measured display-data variation and write time for nine configurations of the fabricated proposed pixel designs, denoted as P-1 to P-9. Each configuration utilizes a different capacitance setting with $C_{s t}=200 \mathrm{fF}$. The device dimension of both $M_{3}$ and $M_{4}$ for all nine configuration is $8 \mu \mathrm{m} / 8 \mu \mathrm{m}$. Similar to Table II, the reported display-data variation and write time is a ratio compared to that of the typical design using T-1 configuration.

As the result shows, the reported display-data variation and write time fit our analysis and simulation results. Basically, a larger $C_{3} / C_{s t}$ can result in a lower display-data variation and a shorter write time before reaching its optimal point $\left(C_{3} / C_{s t}=\right.$ 0.86 in our simulation). Also, a larger $C_{2} /\left(C_{1}+C_{2}\right)$ can always 
TABLE III

MEASUREMENT RESULTS FOR NINE DIFFERENT CONFIGURATIONS OF THE PROPOSED PIXEL DESIGN

\begin{tabular}{|c|c|c|c|c|c|}
\hline \multicolumn{6}{|c|}{ Proposed new design $\left(C_{s t}=200 f F\right)$} \\
\hline Config. & $\begin{array}{c}(\mathrm{W} / \mathrm{L}) \\
\text { of } M_{3,4}\end{array}$ & $\frac{C_{3}}{C_{s t}}$ & $\frac{C_{2}}{\left(C_{1}+C_{2}\right)}$ & $\frac{\Delta V_{P_{0}}}{\Delta V_{P_{2}}}$ & $\frac{W T_{\text {pro }}}{W T_{t y p}}$ \\
\hline P-1 & \multirow{9}{*}{$(8 / 8)$} & $40 \%$ & $10 \%$ & 1.86 & 1.51 \\
\hline $\mathrm{P}-2$ & & $40 \%$ & $20 \%$ & 1.45 & 1.40 \\
\hline $\mathrm{P}-3$ & & $40 \%$ & $30 \%$ & 1.34 & 1.33 \\
\hline $\mathrm{P}-4$ & & $50 \%$ & $10 \%$ & 2.24 & 1.40 \\
\hline $\mathrm{P}-5$ & & $50 \%$ & $20 \%$ & 1.97 & 1.36 \\
\hline P-6 & & $50 \%$ & $30 \%$ & 1.89 & 1.26 \\
\hline P-7 & & $60 \%$ & $10 \%$ & 4.06 & 1.36 \\
\hline $\mathrm{P}-8$ & & $60 \%$ & $20 \%$ & 2.96 & 1.29 \\
\hline P-9 & & $60 \%$ & $30 \%$ & 2.21 & 1.22 \\
\hline
\end{tabular}

result in a shorter write time. Compared to that of the typical design $\mathrm{T}-1$, the best improvement on display-data variation among the nine fabricated proposed designs is $4.06 \times$ with only $1.36 \times$ write time. This result again validates the effectiveness of the proposed pixel design based on real fabricated TFT circuits.

\section{CONCLUSION}

In this paper, we have introduced a novel pixel design to reduce the data variation caused by the leakage current of nc-Si TFT. We first theoretically analyzed the trend of its worst-case data variation and its worst-case write time. Next, several simulations associated with different capacitance settings were conducted to verify the correctness of our analysis. As the result shows, the proposed pixel design can achieve a $5.55 \times$ reduction on its data variation while requiring only $1.15 \times$ write time when compared to the typical pixel design. The resulting contrast resolution can also be improved by two bits. In addition, the area overhead of the proposed pixel design can be effectively limited by the layout techniques, and thus the proposed design can still provide an aperture ratio over $40 \%$. We further discussed the proper setting of the turn-off voltage at Scan_line and the impact of the coupling effect on the proposed pixel design. At last, we reported the measurement results based on the PECVD-based bottom-gate nc-Si-TFT technology developed by ITRI [8].

\section{ACKNOWLEDGMENT}

The authors would like to thank DTC, ITRI for discussion and assistance with fabrication.

\section{REFERENCES}

[1] K. S. Girotra, Y. M. Choi, B. J. Kim, Y. R. Song, B. Choi, S. H. Yang, S. Kim, and S. Lim, "PECVD-based nanocrystalline-silicon TFT backplanes for large-sized AMOLED," J. SID, vol. 15/2, pp. 113-118, 2007.

[2] T. Arai, N. Morosawa, Y. Hiromasu, K. Hidaka, T. Nakayama, A. Makita, M. Toyota, N. Hayashi, Y. Yoshimura, A. Sato, K. Namekawa, Y. Inagaki, N. Umezu, and K. Tatsuki, "Micro silicon technology for active matrix OLED display," SID, vol. 41.2, pp. 1370-1373, 2007.

[3] M. R. Esmaeili-Rad, A. Sazonov, and A. Nathan, "Analysis of the off curent in nanocrystalline silicon bottom-gate thin-film transistors," $J$. Appl. Phys., vol. 103, p. 074502, 2008.

[4] Y. H. Peng, C. H. Chen, C. C. Chang, Y. S. Lee, T. S. Huang, C. Y. Hou, K. F. Huang, and Y. Y. Tseng, "32-inch LCD TV using conventional PECVD microcrystalline-silicon TFTs," SID, vol. 24.3, pp. 333-336, 2008.
[5] C. H. Lee, D. Striakhilev, S. Tao, and A. Nathan, "Top-gate TFTs using 13.56 MHz PECVD microcrystalline silicon," IEEE Electron Device Lett., vol. 26, no. 9, pp. 637-639, Sep. 2005.

[6] K. Girotra, S. Park, J. M. Huh, C. Hong, K. Park, S. Lee, J. H. Lee, C. Chu, J. Goh, J. Choi, C. Kim, and J. H. Souk, "A 14inch uniform AMOLED display with low cost PECVD based microcrystalline silicon TFT backplanes," SID, vol. P-259L, pp. 1289-1292, 2008.

[7] C. Y. Chang, Y. S. Lee, T. Y. Huang, P. S. Shih, and C. W. Lin, "The effect of microcrystalline silicon film structure on low-high-low band-gap thin film transistor," Mater. Chem. Phys., vol. 62, pp. $153-157,2000$.

[8] Y. Y. Pan, C. M. Yeh, C. N. Li, H. Y. Fang, and Y. C. Chen, "Microcrystalline silicon thin film deposition technology," Ind. Mater., vol. 271, p. 112, 2009.

[9] AU Optronics Corp. (AUO), "AU Optronics successfully develops world's first double sided active matrix OLED," 2004.

[10] W. J. Nam, C. Y. Kim, J. H. Lee, S. G. Park, and M. K. Han, "Highaperture ratio pixel design employing $V_{D D}$ line elimination for reducing OLED current density," SID, vol. 36, pp. 1456-1459, 2005.

[11] C. Adachi, "OLED characteristics at high current density," Lasers Electro-Optics Soc., vol. 2, pp. 594-595, 2003.

[12] A. T. Hatzopoulos, N. Arpatzanis, D. H. Tassis, C. A. Dimitriadis, M. Oudwan, F. Templier, and G. Kamarinos, "Study of the drain leakage current in bottom-gated nanocrystalline silicon thin-film transistors by conduction and low-frequency noise measurements," IEEE Trans. Electron Devices, vol. 54, no. 5, pp. 1076-1082, May 2007.

[13] W. J. Nam, C. Y. Kim, J. H. Lee, S. G. Park, and M. K. Han, "Highaperture ratio AMOLED pixel design employing $V_{D D}$ line elimination for reducing OLED current density," SID, vol. 44.3, pp. 1456-1459, 2005.

[14] W. K. Chen, C. W. Ko, and S. H. Lee, "Bottom and top emission OLED pixel structure," U.S. Patent 7250 728, Jul. 31, 2007.

[15] C. J. Lin, "Top emission active matrix OLED and fabrication method thereof," U.S. Patent 6943 372, Sep. 13, 2005

[16] Y. H. Tai, "Design and operation of TFT-LCD panels," Wu-Nan Culture Enterprise, vol. 3, pp. 97-100, 2006.

[17] D. Dosev, T. Ytterdal, J. Pallares, L. F. Marsal, and B. níguez, "DC SPICE model for nanocrystalline and microcrystalline silicon TFTs," IEEE Trans. Electron Devices, vol. 49, no. 11, pp. 1979-1984, Nov. 2002.

[18] M. S. Shur, M. D. Jacunski, H. C. Slade, A. A. Owusu, T. Ytterdal, and M. Hack, "Spice models for amorphous silicon and polysilicon thin film transistors," Electrochem. Soc. Proc., vol. 242, pp. 96-23, 1996.

[19] P. Servati and A. Nathan, "Modeling of the reverse characteristics of a-Si:H TFTs," IEEE Trans. Electron Devices, vol. 49, no. 5, pp. 812-819, May 2002.

[20] C. W. Han, M. K. Han, M. S. Kim, W. J. Nam, S. J. Bae, K. Y. Kim, and I. J. Chung, "Top-emitting OLED pixel employing cathode-contact structure with a-Si:H thin-film transistors," IEEE Electron. Lett., vol. 43, no. 11, pp. 623-624, Nov. 2007.

[21] M. H. Lu, E. Ma, J. C. Sturm, and S. Wagner, "Amorphous silicon TFT active-matrix OLED pixel," in Proc. IEEE Lasers Electro-Optics Soc. Апnu. Meet., 1998, vol. 1, pp. 130-131.

[22] S. H. K. Park, M. Ryu, C. S. Hwang, S. Yang, C. Byun, J. I. Lee, J. Shin, S. M. Yoon, H. Y. Chu, K. I. Cho, K. Lee, M. S. Oh, and S. $\mathrm{Im}$, "Transparent $\mathrm{ZnO}$ thin film transistor for the application of high aperture ratio bottom emission AM-OLED display," SID, vol. 39, pp. 629-632, 2008.

[23] W. J. Nam, J. H. Lee, C. Y. Kim, H. J. Lee, and M. K. Han, "Highaperture pixel design employing $V_{D D}$ line elimination for active matrix organic light emitting diode display," Japan. J. Appl. Phys., vol. 45, pp. 2433-2436, 2006.

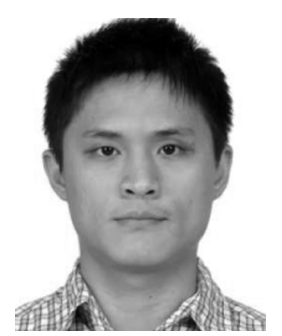

Chen-Wei Lin received the B.S. degree from the Department of Electronics Engineering, National Chiao Tung University, Hsinchu, Taiwan, in 2006 , and the M.S. degree from the Graduate Institute of Electronics Engineering, National Taiwan University, Taipei, Taiwan, in 2007.

His current research interests include thin film transistor (TFT) circuitry design and VLSI design and testing. 


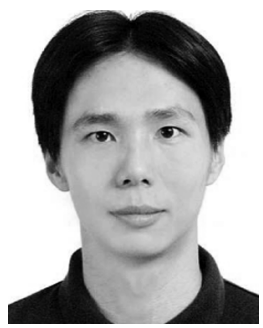

Mango C.-T. Chao (M'07) received the B.S. and M.S. degrees from the Department of Computer and Information Science, National Chiao Tung University, Hsinchu, Taiwan, in 1998 and 2000, respectively, and the Ph.D. degree from the Department of Electrical and Computer Engineering, University of California, Santa Barbara, in 2006.

$\mathrm{He}$ is currently an Assistant Professor with the Department of Electronics Engineering, National Chiao Tung University. His current research interests include VLSI testing, TFT circuitry design, and

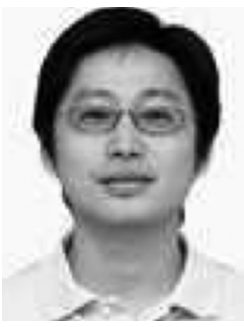

Yen-Shih Huang received the Ph.D. degree from the Department of Materials Science and Engineering, National Tsing Hua University, Hsinchu, Taiwan, in 2002.

He currently works with the Industrial Technology Research Institute, Taiwan. His current research interests include thin film transistor (TFT) devices and AMOLED panel manufacture techniques. 